\title{
A SAURON view of double-barred galaxies
}

\section{Adriana de Lorenzo-Cáceres, Alexandre Vazdekis, Jesús Falcón-Barroso \& Inma Martínez-Valpuesta}

\author{
Instituto de Astrofísica de Canarias \\ C/ Vía Láctea, S/N, E-38205, La Laguna, Tenerife, Spain \\ email: adlcr@iac.es
}

\section{Double bars and the $\sigma$-hollows}

Double bars might be the key mechanisms to transport gas to the very central regions of galaxies, so double-barred galaxies are key objects to better understand the galaxy formation and evolution scenarios. In order to disentangle the role of double bars in the galaxy build up, we are performing a whole kinematical and stellar population analysis of these objects from high $\mathrm{S} / \mathrm{N}$ spectroscopic data.

Four double-barred galaxies were observed with the integral-field spectrograph SAURON at the William Herschel Telescope (La Palma, Spain). The stellar kinematical analysis revealed the presence of the $\sigma$-hollows, i.e. decreases of the velocity dispersion values at the ends of the inner bars (de Lorenzo-Cáceres et al. 2008). These $\sigma$-hollows seem to be the result of the contrast between the high $\sigma$ of the bulge and the low $\sigma$ of the inner bar, meaning that inner bars are cold, thin structures. Fig. 1 shows the intensity and $\sigma$ maps for the four objects.

We have investigated the stellar populations that shape these hollows, comparing the results with the same analysis for the bulge and the rest of the galaxy, within the SAURON field-of-view.

\section{The stellar population analysis}

Fig. 1 shows the line-strength maps of $\mathrm{H} \beta$, Fe5015, and $\mathrm{Mg} b$ for the four galaxies, that were computed by using the Lick definitions of the absorption indices. We find no evidence of the $\sigma$-hollows in none of the maps. Only Fe5015 maps seem to show slightly higher values for the regions of the inner bars, whereas $\mathrm{Mg} b$ values increase only at the centers. No structures are found in the $\mathrm{H} \beta$ maps.

Fig. 2 shows the age indicator $\mathrm{H} \beta_{o}$ (Cervantes \& Vazdekis, 2009) vs. the metallicity indicators $\mathrm{Mg} b, \mathrm{Fe} 5015$ and [MgFe50]. Data points represent a stacked spectrum for each region (center, $\sigma$-hollows and the rest of the field-of-view) of each galaxy. The grids were obtained by using the Vazdekis et al. (2010) stellar population models. Models and data were previously degraded to the maximum value of $\sigma(180 \mathrm{~km} / \mathrm{s})$. The four doublebarred galaxies show an increase of the metallicity values towards the centers, where there are also hints of slightly younger ages. The regions of the $\sigma$-hollows do not show any unexpected population, what supports the idea of their purely dynamical origin.

\section{References}

Cervantes, J. L. \& Vazdekis, A. 2009, MNRAS, 392, 691

de Lorenzo-Cáceres, A., Falcón-Barroso, J., Vazdekis, A., \& Martínez-Valpuesta, I. 2008, ApJ (Letters), 684, L86

Vazdekis, A., et al. 2010, MNRAS, in press 

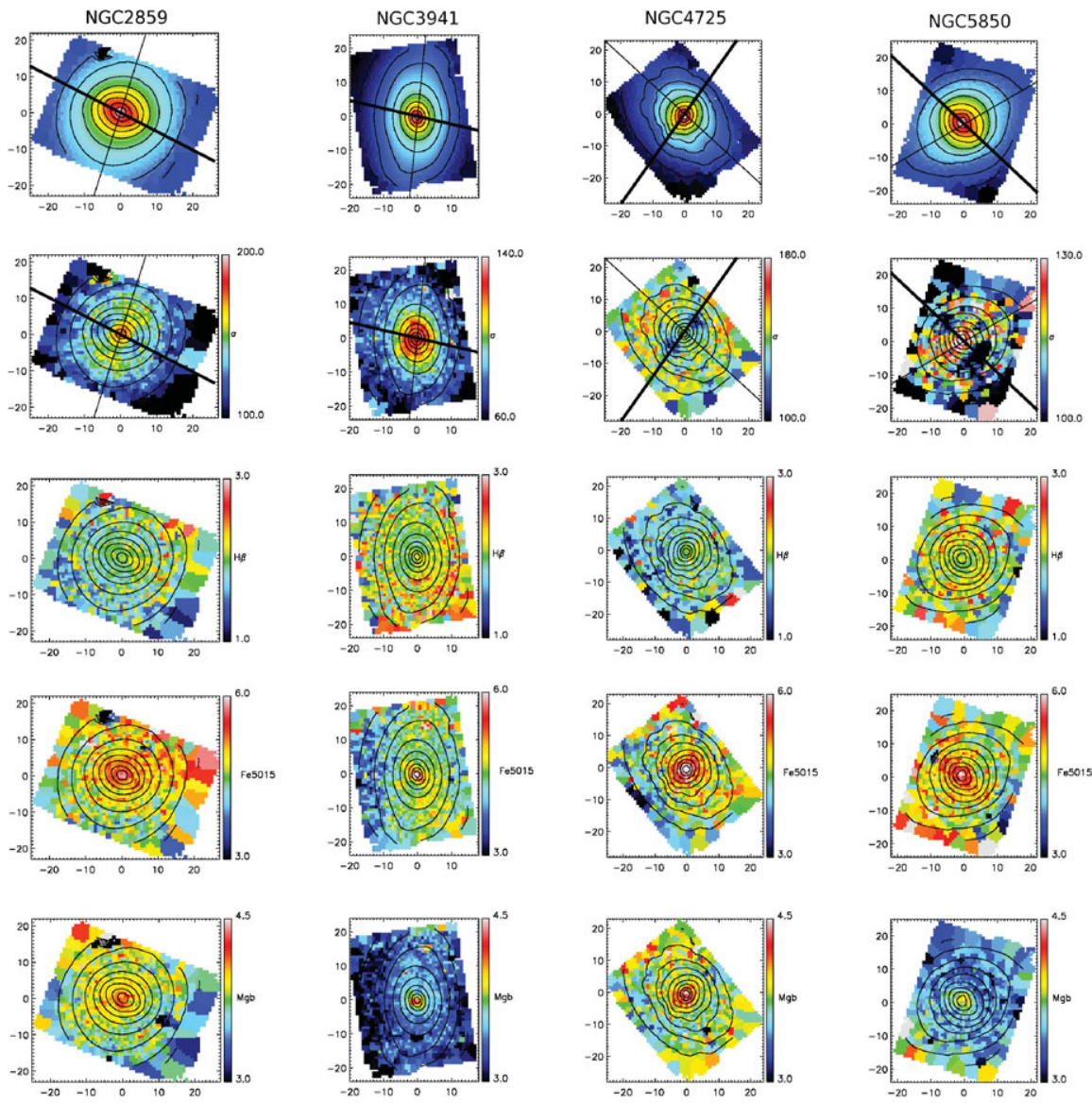

Figure 1. Intensity, velocity dispersion and line-strength $(\mathrm{H} \beta, \mathrm{Fe} 5015$ and $\mathrm{Mg} b)$ maps for the four double-barred galaxies observed with SAURON@WHT. We have overplotted the isodensity contours and, for the intensity and the velocity dispersion maps, the directions of the inner (thick line) and outer (thin line) bars. Spectra reach $\mathrm{S} / \mathrm{N} \sim 300$ in the central regions.
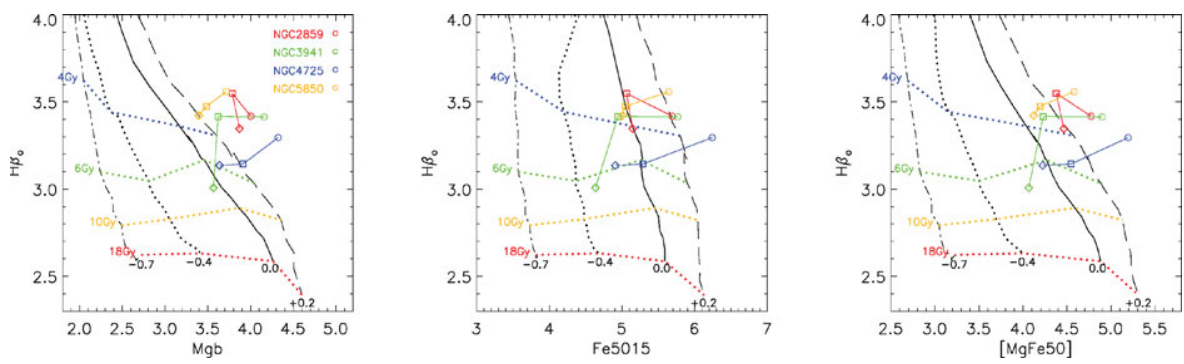

Figure 2. $\mathrm{H} \beta_{o}$ (Cervantes \& Vazdekis, 2009) vs. the metallicity indicators $\mathrm{Mg} b, \mathrm{Fe} 5015$ and $[\mathrm{MgFe} 50]$ (defined as $[\mathrm{MgFe} 50]=\sqrt{M g b * F e 5015}$ ). The grids represent models with different ages (almost horizontal lines) and metallicities (almost vertical lines). The points are the measurements for a stacked spectrum of each interesting region of each galaxy: center (circles), $\sigma$-hollows (squares) and the rest of the field-of-view (diamonds). 